\title{
The trend of morbidity and mortality of Coronavirus disease 2019 under the first-level public health emergency response in China
}

\author{
Jingya $\mathrm{Xu}^{1}$, Xiling $\mathrm{Lin}^{1}$, Xiaowen $\mathrm{Pan}^{1}$, Xin Huang ${ }^{1}$, Ying Chen ${ }^{1}$, Enjie Zhou ${ }^{1}$, Zhaoyuan \\ $\mathrm{Wu}^{1}$, Yuxuan Zou ${ }^{1}$, Feng $\mathrm{Xu}^{1}$, Yuezhong Ren ${ }^{1}$, and Pengfei Shan ${ }^{1}$ \\ ${ }^{1}$ Zhejiang University School of Medicine Second Affiliated Hospital
}

April 28, 2020

\begin{abstract}
The ongoing outbreak of Coronavirus disease 2019 (COVID-19) has already caused a worldwide pandemic, World Health Organization has raised COVID-19 global risk to very high. We aimed to share the Joint Prevention and Control Mechanism taken in Mainland China and evaluate the effectiveness. Data were collected from the daily epidemic reports released by the national and provincial health commissions of China from January 21 to March 26, 2020. Global data were collected from daily situation reports by World Health Organization. Under the first-level public health emergency response, great achievements have been made in controlling the spread of COVID-19 in Mainland China. As of March 26, the daily new deaths were less than 50 in nationwide recently. However, it is still a major challenge worldwide. The comprehensive and powerful control measures taken by Mainland China have proved to be effective and might be applicable to other regions.
\end{abstract}

\section{Introduction}

In late December 2019, clusters of patients with pneumonia of unknown etiology were reported in Wuhan, Hubei Province, China(Lu et al., 2020). The causative agent has been identified as a novel coronavirus, currently known as SARS-CoV-2, which likely originated from bats, according to the gene sequence(Xu et al., 2020; Zhou et al., 2020). The Coronavirus disease 2019 (COVID-19) was of clustering onset(Chen et al., 2020; Li et al., 2020; Yu et al., 2020), and the estimated basic reproductive number R0 ranges from 2.0-4.0(J. T. Wu et al., 2020; Li et al., 2020; Y. Yang et al., 2020; Yu et al., 2020). Complications like acute respiratory distress syndrome (ARDS) and cytokine storm might be the reason of high intensive care admission rate and death(Huang et al., 2020). The outbreak of COVID-19 has led to a public health emergency of international concern declared by World Health Organization (WHO) on January 30, 2020.

China was confronted with multitude challenges in controlling this highly contagious previously unknown disease. The unprecedented pressure posed on the health care system by the exponential patient growth has caused severe shortage of medical resources in Wuhan and even Hubei Province, the epicenter of the outbreak. And coincidentally, the virus strike happened around the Chinese New Year, a traditional national holiday when millions of Chinese migrate in the country. The virus thus began to spread across the country.

The first-level response to public health emergency in China was promptly started in the nation. The timely and forceful Joint Prevention and Control Mechanism initiated by the State Council, have effectively curbed the spread of the COVID-19 in Wuhan and all of Mainland China.

As of March 26, 2020, there are globally 509,164 confirmed COVID-19 cases (46,484 new) in 199 countries/ territories, with 54 news in Mainland China and 46,367 news outside of China. WHO reported that the outbreak of COVID-19 has become a global pandemic, and in fact, COVID-19 is accelerating its spread 
around the world. Although countries face different stages of epidemic and have different levels of health care, the world is facing the same challenges. This study aimed to explore the change patterns of prevalence and death toll in Mainland China, and more important, to share the measures and China's experience to contain the COVID-19.

\section{Materials and methods}

\section{Measures of Joint Prevention and Control for COVID-19 taken by China}

At the early stage of COVID-19 outbreak in Wuhan, the communist party of China (CPC) Central Committee and the State Council launched the national emergency response. Timeline of fighting against SARS-CoV-2 outbreak was described in Figure 1. A central leadership team was established for Epidemic Response and the Joint Prevention and Control Mechanism was determined by the State Council. Control of the COVID19 outbreak was given the top priority of government at all levels. National measures against COVID-19 could be summarized as blow but not limited to Figure 2 .

\section{Enhancing international cooperation}

China has been sharing the latest updates of the epidemic, scientific research achievements and successful experience with the world since the outbreak. For example, the nucleic acid sequence data of the novel coronavirus was shared with the WHO, so were the results of related randomized clinical trials. Academician Nanshan Zhong, was recently invited to Europe to communicate his experience of the prevention and control of the epidemic. Moreover, the Chinese government has been also donating test kits to other countries, when donations from the government and people of other countries, such as face masks, protective clothing, and etc., were continuously sent to China.

\section{Forming leading groups of COVID-10 response in central and local governments}

After the outbreak, Chinese President Jinping Xi personally directed and deployed the prevention and control of the disease. A leading group under the leadership of the Standing Committee of the Political Bureau of the CPC Central Committee was formed to deal with the epidemic, with Chinese Premier Keqiang Li as the head. The leading group is fully responsible for the joint prevention and control of COVID-19 throughout China. Local governments and communities also set up leading groups to response the epidemic.

\section{Launching first-level response to a major public health emergency in 31 provinces}

January 15, the Chinese CDC announced a national-level emergency response. January 20, the National Health Commission issued the Guideline for the Prevention, Diagnosis and Treatment of COVID-19 that has been updated to the 7th version until recently. COVID-19 was classified by the commission as a Category B infectious disease but should be controlled with Category A's prevention and control methods under the Law of the People's Republic of China on the Prevention and Treatment of Infectious Diseases. Since then, number of confirmed cases in each province has been reported on a daily basis by the National Health Commission.

By January 29, all Chinese provinces have declared first-level response to a major public health emergency. The Chinese New Year holiday was extended; people from high-endemic areas and non-urgent workers were encouraged to delay their return; public places, commercial markets and factories were closed; people were asked to restrain their outdoor activities; traffic between communities and villages were shut down to prevent unnecessary visits. Furthermore, the most stringent control on wildlife selling has been implemented, banning all forms of wildlife trade or transportation.

\section{Ensuring the supply of medical resources}

In order to alleviate the shortage of medical resources in Hubei, there has been 380 medical teams comprising of more than 42,000 doctors, nurses, infection control practitioners and public health experts from all over China to provide their help in Wuhan as of February 25. What's more, each province has been paired with one prefecture-level city of Hubei other than Wuhan to deliver medical aid and vital personal protective 
equipment to the people of the whole province. In addition, with the help of telemedicine and artificial intelligence, hundreds of thousands of doctors across the country have been conducting online consultations, greatly easing the pressure on the overall Chinese and Wuhan healthcare system and effectively reducing the number of walk-in patients and thus their risks of infection exposure.

On the other hand, the Leishenshan Hospital and Huoshenshan Hospital, Wuhan versions of Beijing Xiaotangshan Hospital in the fight against SARS in 2003, were established in Wuhan, the design and construction of which was completed in 12 days and 9 days respectively, providing more than 14,000 isolation beds. Another 30,000 beds for mild patients were also managed in mobile cabin hospitals that were remodeled day and night. Moreover, to deal with potential increasing patient volume in other provinces, hospitals for fever patients were designated and regular wards were transformed into isolation wards. In addition to covering all the individual cost of treatment, the government also fully supports the acceleration of basic research and clinical trials of COVID-19, and its vaccine development.

\section{Detecting possible infections early}

Since February 6, Wuhan government has worked tremendously on household-to-household daily temperature checks of all of its residents, for the sake of early detection and early treatment. In order to avoid falsenegative diagnosis, Hubei Province classifies suspected cases with radiographic features of pneumonia, but with negative nucleic acid test results as confirmed cases. In places outside Hubei, people returning from Hubei Province are accurately identified, and home quarantined for 14 days during which temperature check and health condition have to be daily reported. Anyone with fever and/or respiratory discomfort is required to report immediately to local health officials and would be transferred to the fever clinic of special hospitals by ambulance right away.

\section{Protecting vulnerable people}

Individual protection measures includes reducing unnecessary social contact, wearing facemask, washing hands and checking body temperature every day. As for health care workers, hospitals should strictly control nosocomial infection by using different levels of protective equipment in different treatment areas. It is also suggested that the mental health of front-line medical staff shall need proper attention and long hours of heavy workload shall be avoided.

\section{Strengthening the security of supplies}

Chinese government promptly organized enterprises to resume production of household goods and medical supplies (including medical protective clothing, N95 masks, medical goggles, negative pressure ambulances and related drugs) to guarantee domestic supply. Also, all national customs have been fully committed to ensuring the rapid clearance of and the exemption of import duties on materials for epidemic prevention and control. In addition, Chinese government urges all drugstores to open normally without raising prices. In sealed-off communities, food are delivered to home by neighborhood committee. Meanwhile, National Grid has promised not to power off Wuhan residents in arrears with their electricity bills.

\section{Information technology assisting prevention and control}

Information technology such as big data and artificial intelligence, has been widely involved in multiple fields. For example, information released by transport authorities help local governments track and manage priority populations. Passengers on the same flight or train compartment with people later confirmed as COVID-19 patients would be picked out, registered in the community's monitoring list and asked to quarantine. Health code are created by tracking individual's travel information (like whether one has been to high-risk areas), people without qualified health code would be advised to restrain outdoor activities. Zhejiang Province was also the first to present the epidemic risk of each area to the public on a five-color map, each color corresponding to a risk level.

On the other hand, information technology keeps people connected in a more precise and effective way amid the outbreak. Online classes and online office reduce direct contact while maintain the quality of teaching 
and work; authoritative and reliable information such as medical guidance can only be found on government website. The use of information technology makes the disease control more scientific, precise and effective.

\section{Data sources}

Data were collected from the daily epidemic reports released by Chinese national and provincial health commissions, including numbers of confirmed, suspected and recovered cases and death toll, etc. (http://www.nhc.gov.cn/xcs/yqth gzbd.shtml). The definition of confirmed case and severe pneumonia is based on the diagnosis and treatment scheme released by the National Health Commission of China. Interested data were collected from January 21 to March 26 (reported on March 27), 2020 in 34 provinces of China including: 1) new, daily and cumulative confirmed cases; 2) new and cumulative death cases; 3) new and cumulative recovered cases; 4) daily severe pneumonia. Outside China, data were collected from daily situation reports by World Health Organization including cumulative confirmed and death cases (https://www.who.int/emergencies/diseases/novelcoronavirus-2019/situation-reports/).

\section{Statistical analysis}

Case fatality ratio (CFR) was calculated by dividing the number of cumulative deaths by the number of cumulative confirmed cases in a time. All statistical analyses were conducted using Prism software (version 8, GraphPad). Data collection and analysis were considered as part of a continuing public health outbreak investigation and exempt from institutional review board approval.

\section{Results}

\section{The trend of COVID-19 in Wuhan city and Hubei Province}

As of March 26, 2020, the cumulative confirmed COVID-19 cases are 50,006 in Wuhan and 67,801 in Hubei. The cumulative confirmed cases and death toll in Wuhan accounted for $73.75 \%$ and $79.87 \%$ of that in Hubei, respectively. Thus the curve of Wuhan is similar to that of Hubei (Figure 3). The daily number of new confirmed cases significantly increased between Jan 21 and Feb 4, 2020, then plateaued between February 4 and February 11. A peak of 14,840 daily increment in Hubei was reported on February 12. The daily number of new confirmed cases continued to decrease in recent days. On March 26, 2020, the daily new confirmed cases was 0 in Hubei. In terms of death, the death toll increment in Hubei peaked and plateaued between February 12 and February 23, and dropped markedly later. Recently the daily new deaths were less than 10 in nationwide. The number of severe pneumonia cases in Hubei continued to decrease significantly. The case fatality ratio (CFR) was $5.07 \%$ in Wuhan and $4.68 \%$ in Hubei.

\section{The trend of COVID-19 outbreak outside Hubei Province in Mainland China}

The trend of COVID-19 outbreak outside Hubei Province in Mainland China was quite different from that in Wuhan or Hubei due to timely intervention measures. As of March 26, 2020, the numbers of cumulative confirmed and death cases were 13,540 and 118 respectively outside Hubei. The daily number of new confirmed case outside Hubei reached a peak of 880 on February 3 and decreased over time. Recently, the daily numbers of new confirmed cases are very low and mostly cases contracted outside China. The overall death toll outside Hubei is much lower with a CFR of $0.87 \%$ as of March 26, 2020. It is gratifying to see that daily number of recovered cases is increasing rapidly both inside and outside Hubei with the latest recover rate as $91.05 \%$ and $94.88 \%$ as of March 26, 2020, respectively. It is worth noting that there were up to 19 provinces reporting zero new infections, and almost all provinces with new cases are imported cases as of March 26, 2020, especially in highly prevalent provinces like Hunan which was no new infections over 27 days.

\section{Current geographic distribution of COVID-19 in and outside China}

Figure 4A depicted distribution of confirmed and death cases of COVID-19 in China as of March 26, 2020. Hubei accounted for the majority of the total confirmed $(83.36 \%)$ and death $(96.42 \%)$ number. The highest confirmed case was in Hubei $(67,801)$; clusters are notable in neighboring provinces of Henan $(1,275)$ and Hunan $(1,018)$, as well as in Guangdong $(1,456)$ and Zhejiang $(1,247)$ province. Despite the fact that almost 
all death cases were in Hubei $(3,174)$, Henan (22) and Heilongjiang (13) were on the top as well. Outside Hubei, the death toll is indeed low and some provinces reported none.

Outside of China, there were 427,086 confirmed cases reported in 198 countries and the total death number were 20,037 as of March 26, 2020. The total daily new confirmed case outsides China exceeded those in China on February 26. The highest confirmed case was in Italy $(80,539)$, followed by U.S.A $(68,334)$ and Spain $(56,188)$. The top three death toll were in in Italy $(8,165)$, Spain $(4,089)$ and Iran $(2,234)$. The overall CFR varies in countries with Italy $(10.14 \%)$, Iran $(7.60 \%)$ and Spain $(7.28 \%)$ to be in detail.

\section{Discussion}

In the battle against COVID-19, China has rolled out perhaps the most ambitious, agile and aggressive disease containment effort in history (World Health Organization. Report of the WHO-China Joint Mission on Coronavirus Disease 2019 (COVID-19). 2020). The rapid spread and the course of this rapidly escalating and deadly epidemic has been changed under the China's powerful and scientific Joint Prevention and Control Mechanism. The effective control of COVID-19 in China provides at least two prevention and control modes for similar highly contagious diseases, one being the control mode in Wuhan, the epicenter of the outbreak, and the other being the early control mode in regions outside Hubei Province in China.

Consistent with our knowledge about other coronaviruses, SARS-CoV-2 is mainly transmitted via respiratory droplets and contact. In China, interpersonal transmission of COVID-19 occurs mainly in families (Chen et al., 2020; Li et al., 2020; Yu et al., 2020), and the relative high basic reproductive number R0 (J. T. Wu et al., 2020; Li et al., 2020; Y. Yang et al., 2020; Yu et al., 2020) may account for the nationwide outbreak. All populations are susceptible to SARS-CoV-2, including children and infants, and elderly men with underlying diseases are prone to develop severe cases (Chen et al., 2020; D. Wang et al., 2020; Huang et al., 2020; Liang et al., 2020; Xu X , Wu X, Jiang X, 2020; Z. Wu and McGoogan, 2020). Despite implementing comprehensive controls in a shorter period of time, the number of confirmed cases of COVID-19 still far exceeds SARS (8,422 cases), suggesting that SARS-CoV-2 may be more contagious than SARS-CoV. Although the case fatality rate (CFR) is much lower than SARS (9\%) and MERS (36\%) (Su et al., 2016), COVID-19 results in a higher overall death toll due to the large number of cases. It is worth noting that it is widely reported that asymptomatic infected people are also contagious (Bai et al., 2020; Lirong Zou et al., 2020; Pan et al., 2020; Rothe et al., 2020; Sebastian Hoehl et al., 2020; Shi et al., 2020; Yu et al., 2020), which is different from SARS-CoV (Zeng et al., 2009), greatly enhancing the difficulty of outbreak control (Fraser et al., 2004; Peak et al., 2017).

The exponential growth of patients nearly crashed down the health care system of Wuhan, even Hubei Province. In order to alleviate the shortage of medical resources in Hubei, as of February 25, 380 medical teams comprising of more than 42,000 health workers from all over China have been providing their help in Wuhan. In addition to increasing isolation wards in existing hospitals and transforming general hospital into infectious disease hospital, Wuhan established the Leishenshan Hospital and Huoshenshan Hospital as designated hospitals of COVID-19. A dozen mobile cabin hospitals were also built overnight to treat mild patients. Meanwhile, apart from lockdown of city and traffic quarantine, Wuhan government has also worked tremendously on household-to-household daily temperature checks of all of its residents and strictly controlled communities. As promising antiviral drugs, Remdesivir and chloroquine prove to effectively inhibit SARS-CoV-2 in vitro (M. Wang et al., 2020), several randomized controlled trials are already underway (https://clinicaltrials.gov/ct2/show/NCT04252664?term=remdesivir\&draw=2\&rank=2. https://clinicaltrials.gov/ct2/show/NCT04257656?term=remdesivir\&draw $=2 \&$ rank=1. https://clinicaltrials.gov/ct2/show/ $19+$ Treatment+Trial\&draw $=2 \& \mathrm{rank}=1$.), and the successful use of Remdesivir in relieving clinical symptoms in the United States also provides a clinical reference (Holshue et al., 2020). In addition, Chinese herbal medicine has been proven to greatly relieve patients' condition. The combination of treatment and public health intervention has shown results in recent days. According to the prediction model of Nanshan Zhong 's team (Z. Yang et al., 2020), lifting the Hubei quarantine (such as cancelling lockdown of Wuhan and surrounding cities, returning to normal Spring Festival travel rush) would result in a second epidemic peak in Hubei Province in mid-March and extend the epidemic to late April. But in fact, the number of newly 
confirmed case in Wuhan has changed from several thousand to 0 on March 18, indicating that the outbreak in Wuhan has been well controlled. Wuhan's experience in control and treatment provides a good reference for other countries/regions with COVID-19 outbreak.

Outside Hubei, China has employed another model of prevention and control, because, unlike Hubei, the other provinces are at the early stage of outbreak. Despite sending medical staff and personal protective equipment to Hubei, many provinces were actually confronted with tough situation. The official classification of COVID-19 as a Category B infectious disease with the prevention and control methods of Category A, has marked the transition from local-level to central level of disease prevention and control. By January 29, all provinces in Mainland China have launched first-level response to a major public health emergency. The aim of all enforced public health interventions was to isolate, diagnosis, and treat people coming back from Hubei Province as early, quarantine those having contacts with confirmed case, and reduce the possibility of secondary transmission in other provinces. Take Guangdong and Zhejiang Province for example, as popular destinations for migrant workers, the two provinces effectively prevent the spread of COVID-19 by continuous early detection and early isolation of Hubei-related residents. Nationally, the number of newly confirmed cases outside Hubei increased rapidly from January 20 to February 2, reached a peak on February 3. Since then, it has maintained a steady downward trend, with only an exception peak on February 20.

Although China has made great achievements in this anti-virus combat, the epidemic is still surging worldwide and involving more and more countries (https://www.who.int/docs/default-source/coronaviruse/situationreports/20200319-sitrep-59-covid-19.pdf?sfvrsn=c3dcdef9_2.). WHO has raised COVID-19 global risk to very high on March 13. And dramatically, the number of confirmed cases worldwide took over three months to reach the first 100,000 confirmed cases, and only 12 days to reach the next 100,000, even grow exponentially around the world in recent days. In the absence of a specific antiviral drug or vaccine, other countries could follow China's example to prevent a global outbreak. At the same time, China should stay alert. The focus should be shifted from preventing the spread of domestic epidemics to the importation of international cases. Only when the government pays enough attention together with global effort can epidemic prevention work be better carried out. What's more, Tie Song, deputy director of the Guangdong Provincial CDC, said 14\% of discharged patients in Guangdong returned positive nucleic acid results (http://www.scio.gov.cn/xwfbh/gssxwfbh/xwfbh/guangdong/Document/1674339/1674339.htm.). JAMA also reported that the pharyngeal swab nucleic acid results of 4 medical staff who have been cured turned out to be re-positive (Lan et al., 2020). Some experts believe that the virus in the patient has always been positive, the difference is whether it was successfully detected. Therefore, it is of high priority of other countries to confirm the proportion of discharged patients with positive nucleic acid results and whether they are contagious. Whether it is necessary to use more sensitive nucleic acid kits, increase the location of samples, adopt more strict discharge standards or post-discharge isolation measures, all are the focus of our future work.

In conclusion, China has made a great achievement in control the spread of COVID-19 through the enforced nationwide intervention even suspension of normal social and economic operations and sacrifice by China and its people in both human and material terms. However, there are still many challenges and a lot of work need to do to curb the new disease since outbreak occurred internationally now, such as explore the source of infection, transmissibility, pathogenesis and virulence of the virus, risk factors for infection and disease progression, surveillance, diagnostics, the effective drugs and vaccine development. China has shared successful model of joint prevention and management experience against COVID-19 with international community. In face this previous unknown disease, all countries need to unite, learn from the excellent prevention and control model, adopt the method suitable for their own countries and regions, to control the new pneumonia.

\section{Acknowledgement}

We are very grateful to the people of China and the world for their tremendous efforts in fighting against COVID-19. We are very grateful to the health workers and people involved working day and night without fear of death. Without the concerted efforts of all the people, COVID-19 in China could not be possibly 
constrained in 2 months. Special thanks also go to all levels of Chinese health commissions and WHO for making the data public.

\section{Conflict of interests}

PFS has received research grants from the National Natural Science Foundation of China (81670744, 81870564) and Science Technology Department of Zhejiang Province of China (No. 2017C33037).

\section{ETHICAL APPROVAL}

This study did not involve animal experiments besides the fae-

cal sampling of diarrhoea dairy calves that visited farm for clinical

treatment.

\section{Ethical approval}

This study did not involve any ethical approval.

\section{References}

Bai, Y., L. Yao, T. Wei, F. Tian, D.-Y. Jin, L. Chen, \& M. Wang. (2020). Presumed Asymptomatic Carrier Transmission of COVID-19.JAMA https://doi.org/10.1001/jama.2020.2565.

Chen, N., M. Zhou, X. Dong, J. Qu, F. Gong, Y. Han, ...L. Zhang. (2020). Epidemiological and clinical characteristics of 99 cases of 2019 novel coronavirus pneumonia in Wuhan, China: a descriptive study.Lancet 395 , 507-513, https://doi.org/10.1016/S0140-6736(20)30211-7.

Fraser, C., S. Riley, R.M. Anderson, \& N.M. Ferguson. (2004). Factors that make an infectious disease outbreak controllable. Proc. Natl. Acad. Sci. U. S. A. 101, 6146-6151, https://doi.org/10.1073/pnas.0307506101.

Holshue, M.L., C. DeBolt, S. Lindquist, K.H. Lofy, J. Wiesman, H. Bruce, .. S.K. Pillai. (2020). First Case of 2019 Novel Coronavirus in the United States. N. Engl. J. Med. 382, 929-936, https://doi.org/10.1056/nejmoa2001191.

Huang, C., Y. Wang, X. Li, L. Ren, J. Zhao, Y. Hu, ... B. Cao. (2020). Clinical features of patients infected with 2019 novel coronavirus in Wuhan, China. Lancet 395 , 497-506, https://doi.org/10.1016/S01406736(20)30183-5.

Lan, L., D. Xu, G. Ye, C. Xia, S. Wang, Y. Li, \& H. Xu. (2020). Positive RT-PCR Test Results in Patients Recovered From COVID-19.JAMA https://doi.org/10.1001/jama.2020.2783.

Li, Q., X. Guan, P. Wu, X. Wang, L. Zhou, Y. Tong, ... Z. Feng, 2020: Early Transmission Dynamics in Wuhan, China, of Novel Coronavirus-Infected Pneumonia. N. Engl. J. Med. 1-9, https://doi.org/10.1056/nejmoa2001316.

Liang, W., W. Guan, R. Chen, W. Wang, J. Li, K. Xu, ... J. He (2020). Cancer patients in SARS-CoV-2 infection: a nationwide analysis in China. Lancet Oncol. 21, 335-337, https://doi.org/10.1016/s14702045(20)30096-6.

Lirong Zou, Feng Ruan, Mingxing Huang, Lijun Liang, Huitao Huang, Zhongsi Hong, ... Jie Wu (2020). SARS-CoV-2 Viral Load in Upper Respiratory Specimens of Infected Patients. N. Engl. J. Med.382 , $1177-1179$.

Lu, H., C.W. Stratton, \& Y.W. Tang (2020). Outbreak of pneumonia of unknown etiology in Wuhan, China: The mystery and the miracle. J. Med. Virol. 92, 401-402, https://doi.org/10.1002/jmv.25678.

Pan, X., D. Chen, Y. Xia, X. Wu, T. Li, X. Ou, ... J. Liu (2020). Asymptomatic cases in a family cluster with SARS-CoV-2 infection.Lancet Infect. Dis.https://doi.org/10.1016/S1473-3099(20)30114-6.

Peak, C.M., L.M. Childs, Y.H. Grad, \& C.O. Buckee (2017). Comparing nonpharmaceutical interventions for containing emerging epidemics.Proc. Natl. Acad. Sci. U. S. A. 114, 4023-4028, https://doi.org/10.1073/pnas.1616438114. 
Rothe, C., M. Schunk, P. Sothmann, G. Bretzel, G. Froeschl, C. Wallrauch, ... M. Hoelscher (2020). Transmission of 2019-nCoV Infection from an Asymptomatic Contact in Germany. N. Engl. J. Med. 382 , 970-971, https://doi.org/10.1056/nejmc2001468.

Sebastian Hoehl, Holger Rabenau, Annemarie Berger, M. Kortenbusch, Jindrich Cinatl, \&M.S. Denisa Bojkova (2020). Evidence of SARS-CoV-2 Infection in Returning Travelers from Wuhan, China. N. Engl. J. Med. https://doi.org/10.1056/NEJMc2001899.

Shi, H., X. Han, N. Jiang, Y. Cao, O. Alwalid, J. Gu, ... C. Zheng (2020). Radiological findings from 81 patients with COVID-19 pneumonia in Wuhan, China: a descriptive study. Lancet. Infect. Dis.https://doi.org/10.1016/S14733099(20)30086-4.

Su, S., G. Wong, W. Shi, J. Liu, A.C.K. Lai, J. Zhou, ... G.F. Gao (2016). Epidemiology, Genetic Recombination, and Pathogenesis of Coronaviruses. Trends Microbiol. 24, 490-502, https://doi.org/10.1016/j.tim.2016.03.003.

Wang, D., B. Hu, C. Hu, F. Zhu, X. Liu, J. Zhang, .. Z Z. Peng (2020). Clinical Characteristics of 138 Hospitalized Patients with 2019 Novel Coronavirus-Infected Pneumonia in Wuhan, China. JAMA.https://doi.org/10.1001/jama.2020.

Wang, M., R. Cao, L. Zhang, X. Yang, J. Liu, M. Xu, ...G. Xiao (2020). Remdesivir and chloroquine effectively inhibit the recently emerged novel coronavirus (2019-nCoV) in vitro. Cell Res.30 , 269-271, https://doi.org/10.1038/s41422-020-0282-0.

World Health Organization. Report of the WHO-China Joint Mission on Coronavirus Disease 2019 (COVID19). 2020.

Wu, J.T., K. Leung, \& G.M. Leung (2020). Nowcasting and forecasting the potential domestic and international spread of the 2019-nCoV outbreak originating in Wuhan, China: a modelling study. Lancet395 , 689-697 https://doi.org/10.1016/S0140-6736(20)30260-9.

Wu, Z., \& J.M. McGoogan (2020). Characteristics of and Important Lessons From the Coronavirus Disease 2019 (COVID-19) Outbreak in China: Summary of a Report of 72314 Cases From the Chinese Center for Disease Control and Prevention. JAMAhttps://doi.org/10.1001/jama.2020.2648.

Xu, X., P. Chen, J. Wang, J. Feng, H. Zhou, X. Li, ... P. Hao (2020). Evolution of the novel coronavirus from the ongoing Wuhan outbreak and modeling of its spike protein for risk of human transmission. Sci. China Life Sci. 63, 457-460, https://doi.org/10.1007/s11427-020-1637-5.

Xu Xiao-Wei, Wu Xiao-Xin, Jiang Xian-Gao, Xu Kai-Jin, Ying Ling-Jun, Ma Chun-Lian, ... Li Lan-Juan (2020). Clinical findings in a group of patients infected with the 2019 novel coronavirus (SARS-Cov-2) outside of Wuhan, China: retrospective case series. BMJhttps://doi.org/10.1136/bmj.m606.

Yang, Y., Q. Lu, M. Liu, Y. Wang, A. Zhang, N. Jalali, ... L. Fang (2020). Epidemiological and clinical features of the 2019 novel coronavirus outbreak in China. medRxivhttps://doi.org/10.1101/2020.02.10.20021675.

Yang, Z., Z. Zeng, K. Wang, S. Wong, W. Liang, M. Zanin, ... J. He (2020). Modified SEIR and AI prediction of the epidemics trend of COVID-19 in China under public health interventions. https://doi.org/10.21037/jtd.2020.02.64.

Yu, P., J. Zhu, Z. Zhang, Y. Han, \& L. Huang (2020). A familial cluster of infection associated with the 2019 novel coronavirus indicating potential person-to-person transmission during the incubation period.J. Infect. Dis. 1-5, https://doi.org/10.1093/infdis/jiaa077.

Zeng, G., S.Y. Xie, L.I. Qin, \& J.M. Ou (2009). Infectivity of severe acute respiratory syndrome during its incubation period. Biomed. Environ. Sci. 22, 502-510, https://doi.org/10.1016/S0895-3988(10)60008-6.

Zhou, P., X. Yang, X. Wang, B. Hu, L. Zhang, W. Zhang,\& Z. Shi (2020). A pneumonia outbreak associated with a new coronavirus of probable bat origin. Nature 579, 270-273, https://doi.org/10.1038/s41586-0202012-7.

Legend for Figure 1. Timeline of COVID-19 outbreak. 
Legend for Figure 2. Strategies for control COVID-19 in Mainland China

Legend for Figure 3. Changes of COVID-19 in mainland China (including Wuhan, Hubei and outside Hubei in mainland China) . (A) Daily new confirmed cases; (B) Cumulative confirmed cases; (C) Daily new death cases; (D) Cumulative death cases; (E) Daily recovered cases; (F) Cumulative recovered cases; (G) Daily severe pneumonia cases; (H) Daily confirmed cases.

Legend for Figure 4. Distribution of confirmed COVID-19 cases and death in and outside China as of 18 March 2020. The bubble represent the death number of corresponding province or countries
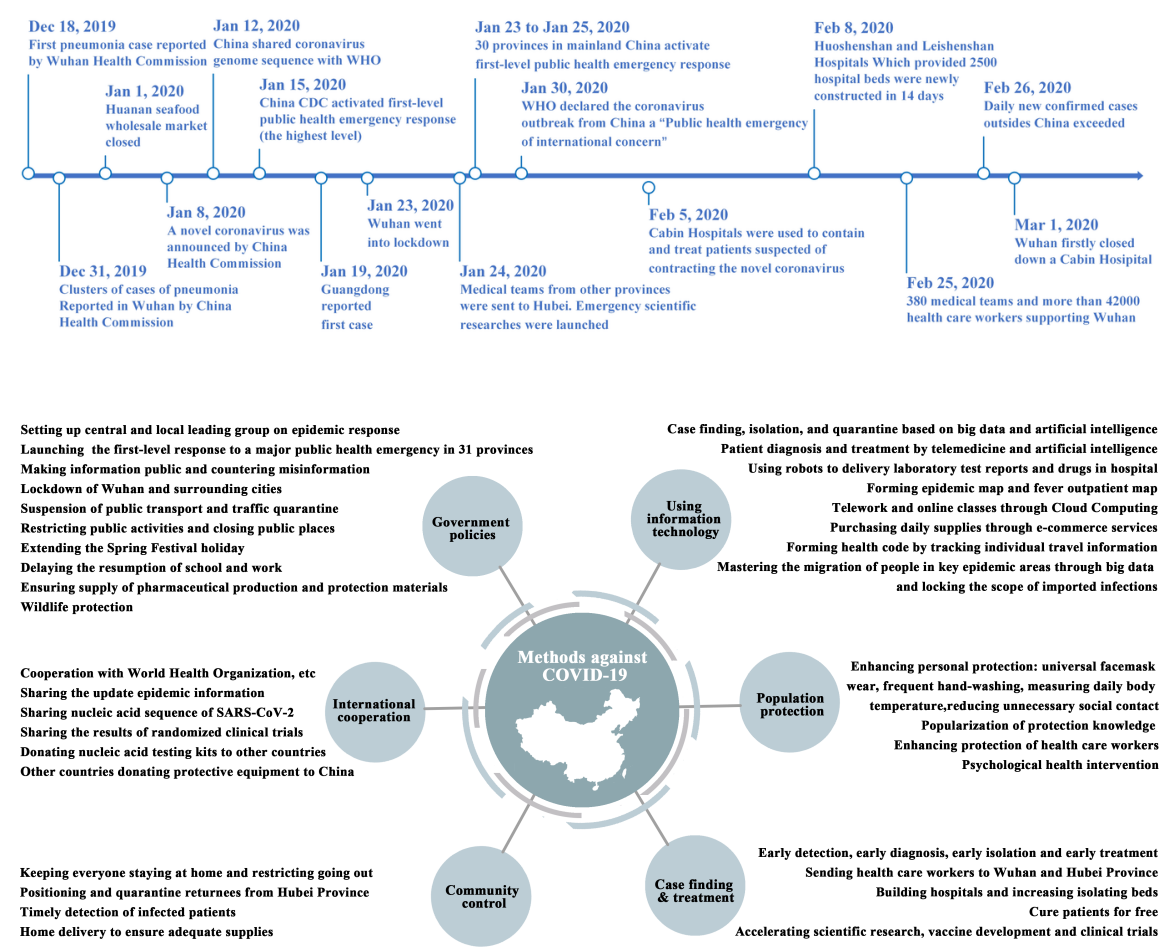
A

Daily new confirmed COVID-19 cases

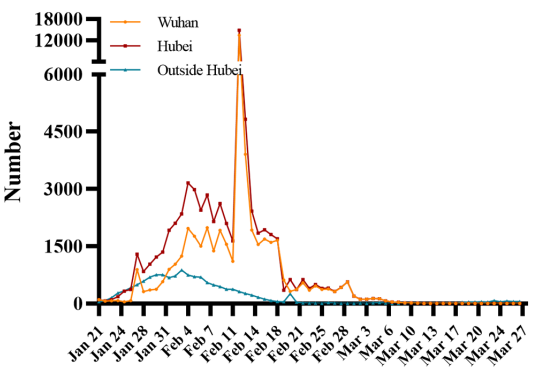

C

Daily new COVID-19 death cases

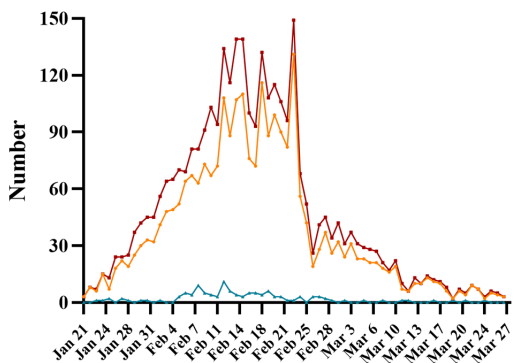

E

Daily new recovered COVID-19 cases

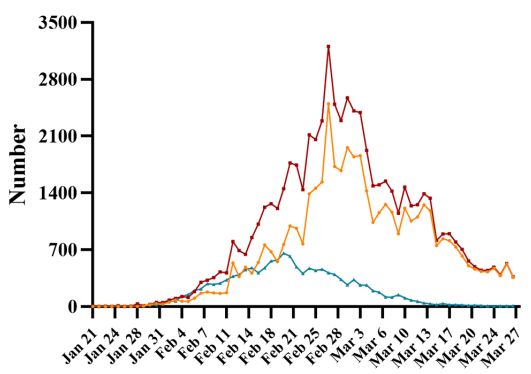

G

Daily severe pneumonia of COVID-19 cases

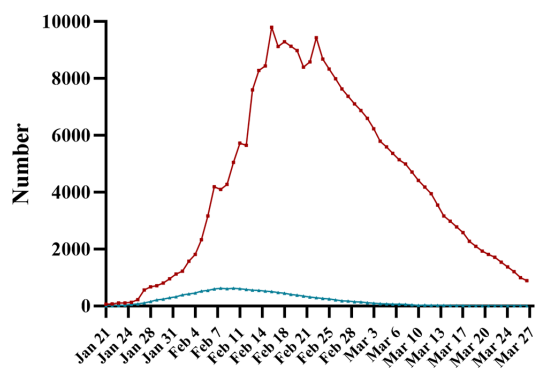

B

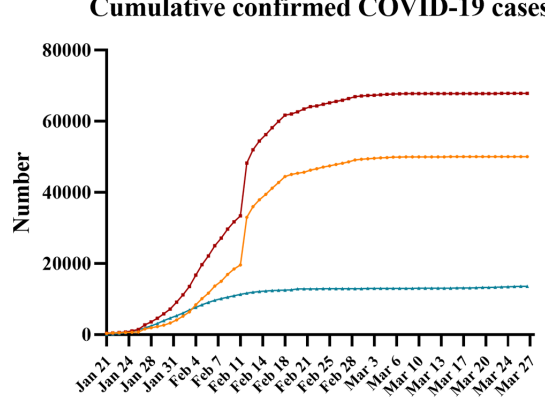

D

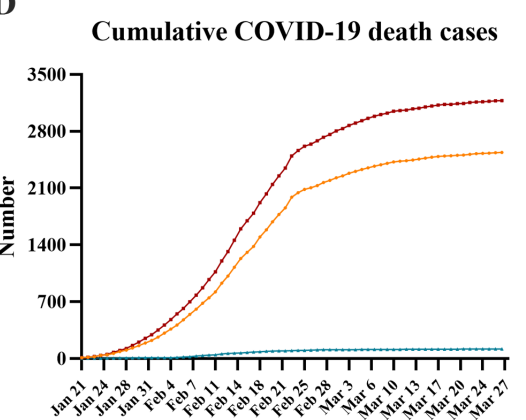

F

Cumulative recovered COVID-19 cases

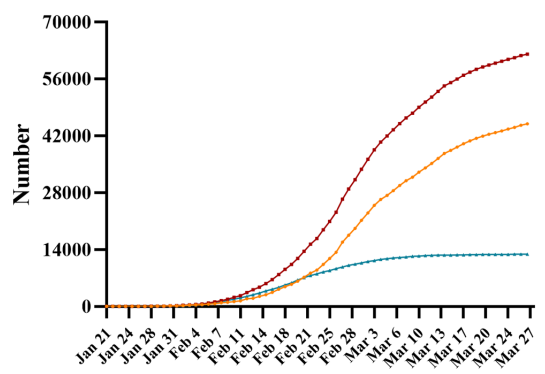

$\mathbf{H}$

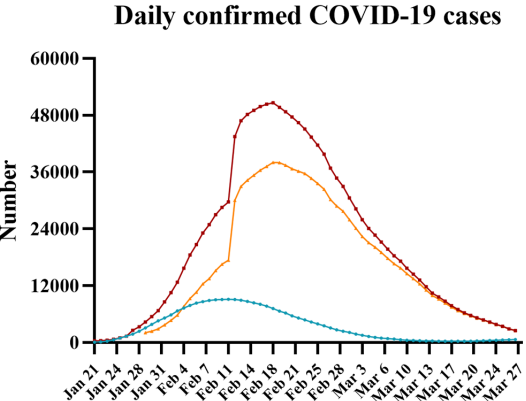


A

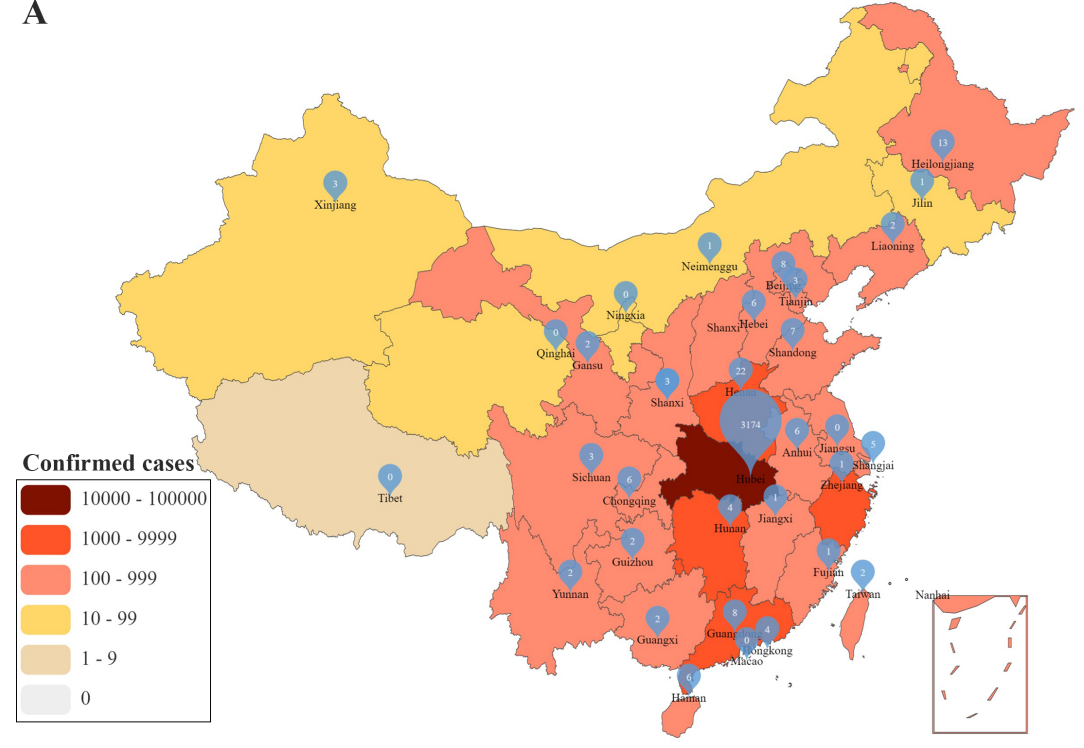

B

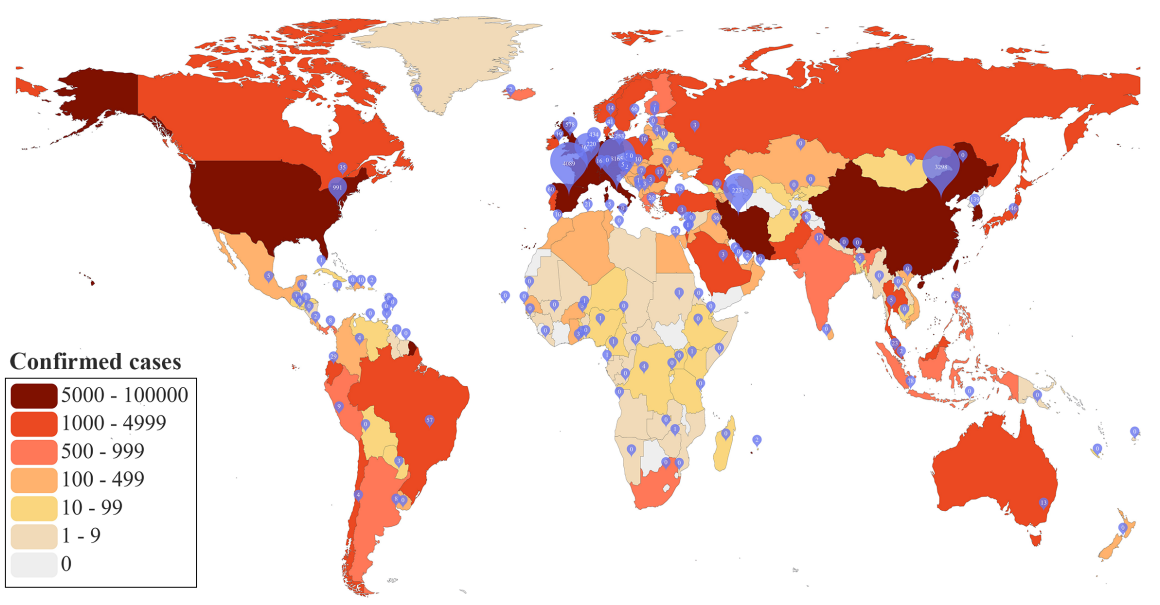

